\title{
Optimal Target Channel Sequence Design for Multiple Spectrum Handoffs in Cognitive Radio Networks
}

\author{
Li-Chun Wang, Fellow, IEEE, Chung-Wei Wang, and Chung-Ju Chang, Fellow, IEEE
}

\begin{abstract}
In this paper, we investigate how to determine an optimal target channel sequence with the minimum cumulative handoff delay in cognitive radio networks. When the secondary user encounters multiple spectrum handoffs during its transmission period, the effects of multiple interruptions from the high-priority primary users and the traffic statistics of both the primary and the secondary users should be incorporated in the design of the optimal target sequence. The optimal target channel sequence can guild the secondary user to change its operating channel when the primary user's interruptions occur. With $M$ candidate channels and $L$ elements in the target channel sequence for spectrum handoffs, the exhaustive search (ES) requires time complexity of $O\left(M^{L}\right)$. In this paper, we propose a dynamic programming (DP) algorithm with time complexity of $O\left(L M^{2}\right)$ to determine an optimal target channel sequence. Furthermore, we propose a greedy algorithm with time complexity of $O(M)$ and prove that the greedy algorithm only requires comparing six target channel sequences. Numerical results show that the low-complexity greedy algorithm can yield similar cumulative handoff delay performance as the optimal DP-based or ES-based algorithms in most cases except when the primary users' service time distributions at their operating channels are different.
\end{abstract}

Index Terms-Cognitive radio, spectrum handoff, spectrum mobility, preemptive priority, queueing theory.

\section{INTRODUCTION}

C OGNITIVE radio (CR) can significantly improve spectrum efficiency by allowing the secondary users to access the under-utilized licensed spectrum of primary users [1]. When a high-priority primary user appears at his/her licensed band, the low-priority secondary users need to vacate the occupied channel, resulting in the spectrum handoff issue. Spectrum handoff procedures are designed to select a target channel for the secondary users to resume the unfinished transmission. Clearly, multiple interruptions from the primary users will result in multiple spectrum handoffs. Hence, a set of target channels, called the target channel sequence, shall be determined for multiple spectrum handoffs during the transmission period of a secondary connection.

Paper approved by O. Simeone, the Editor for Cognitive and Cooperative Communications of the IEEE Communications Society. Manuscript received October 25, 2010; revised July 10, 2011, January 17 and March 24, 2012.

This paper is supported by the National Science Council, Taiwan, under the contract NSC-100-3113-P-009-001. This work was presented in part at the IEEE International Conference on Communications (ICC), 2009.

L.-C. Wang and C.-J. Chang are with the Department of Electrical Engineering, National Chiao Tung University, Hsinchu 300, Taiwan (e-mail: lichun@cc.nctu.edu.tw, cjchang@mail.nctu.edu.tw).

C.-W. Wang is with MStar Semiconductor, Inc., Taipei, Taiwan (e-mail: hyper.cm91g@nctu.edu.tw).

Digital Object Identifier 10.1109/TCOMM.2012.070912.100661
Basically, the target channel selection approaches in spectrum handoff can be categorized into two kinds [2]: (1) ondemand channel selection for the reactive-decision spectrum handoff; (2) predetermined channel selection for the proactivedecision spectrum handoff. The first kind of target channel selection approaches are based on instantaneous spectrum sensing outcomes to find available and good-quality target channels. For this kind of approaches, channel search time is the dominant factor for the cumulative multiple-handoff delay per connection in addition to channel switching time. From the aspect of designing the optimal channel sensing order, [3]-[5] investigated how to minimize the delay of finding available channels for spectrum handoff. Furthermore, the throughput maximization issue for CR networks with adaptive modulation was examined in [6], [7]. An optimal strategy for channel probing and transmission scheduling was proposed in [8]. The methods in [3]-[8] considered the channel search issue in the case of the initial channel access and single spectrum handoff. The impacts of channel search time on the cumulative delay of multiple spectrum handoffs were studied in [9], [10] by using the preemptive resume priority (PRP) M/G/1 queueing approach. In the case of multiple handoffs, it was found that spectrum sensing time in on-demand channel selection can significantly affect spectrum handoff delay.

The second kind of target channel selection approaches are to predetermine the target channels based on the long-term traffic statistics. Because both the transmitter and receiver can know their target channel sequence for future spectrum handoffs before data transmission, achieving a consensus on the target channels between the transmitter and its intended receiver is easier in this approach ${ }^{1}$. Furthermore, the secondary users using this method can immediately change to the predetermined target channel and thus save spectrum sensing time, but the predetermined channel selection approaches need to consider the channel obsolescence issue. Because the predetermined target channel may not be available any more when a spectrum handoff is requested, the secondary user must consider the corresponding waiting time when determining its target channels. In the literature, the predetermined target channel selection methods for spectrum handoffs can be further categorized into two kinds: the probability-based channel

\footnotetext{
${ }^{1}$ The transmitter can notify the corresponding receiver of the selected target channel sequence during connection initialization period, and does not require notifying the corresponding receiver of the selected target channel when interruption occurs.
} 
selection method and Markov decision process.

- In [11]-[13], the probability-based channel selection methods were developed to predetermine the probability that each channel is selected to be the target channel. Based on the predetermined probabilities, the optimal channel hopping sequence was decided in the packet-bypacket or slot-by-slot manners. In [11], [12] the optimal channel hopping sequence was designed for the singlesecondary-user case, while in [13] the similar problem was extended to the multiple-secondary-user scenario. The above approaches for designing the channel hopping sequence were optimal in the sense of maximizing the per-slot throughput. However, the latency issue in spectrum handoff has not been considered yet. It is clear that the cumulative delay per connection due to multiple spectrum handoffs is an important quality of service (QoS) performance measure for CR networks.

- Another kind of target channel selection approaches are to apply the theory of Markov decision process. In [14]-[20], the target channel selection problem in every time slot was modeled as a Markov decision process. According to the channel occupancy state at the current time slot, a decision maker (secondary user) preselected the best action (target channel) to maximize its immediate reward at the next time slot, where the considered reward or the objective function was the expected per-slot throughput [14]-[18], the expected idle period [19], and the expected waiting time [20]. However, these papers only considered the effects of channel usage behaviors of the primary users on the channel occupancy. Thus, it would be interesting to examine how the traffic loads of secondary users affect the target channel selection for spectrum handoff.

In this paper, we formulate an optimization problem for the proactively predetermined target channel selection with the objective of minimizing the cumulative delay per connection for a newly arriving secondary user, while taking into account of the channel switching time and the waiting time resulting from the channel obsolescence effect. To ensure the QoS of the secondary users, the optimal sequence of preselected target channels shall incorporate two important factors: (1) multiple interruptions from the primary users in each secondary users' connection; (2) various arrival rates and service time of both the primary and secondary users. The contributions of this paper can be summarized in the following:

- A dynamic-programming-based algorithm with the time complexity of $O\left(L M^{2}\right)$ is proposed to find an optimal target channel sequence with minimum cumulative spectrum handoff delay, where $L$ and $M$ are the lengths of the target channel sequence and the total number of candidate channels for spectrum handoffs, respectively.

- Furthermore, a sub-optimal but low-complexity greedy algorithm with $O(M)$ time complexity is proposed. We prove that it is only necessary to compare six target channel sequences, and demonstrate that the cumulative multiple-handoff delay of the greedy algorithm is close to that of the optimal dynamic-programming-based algorithm.

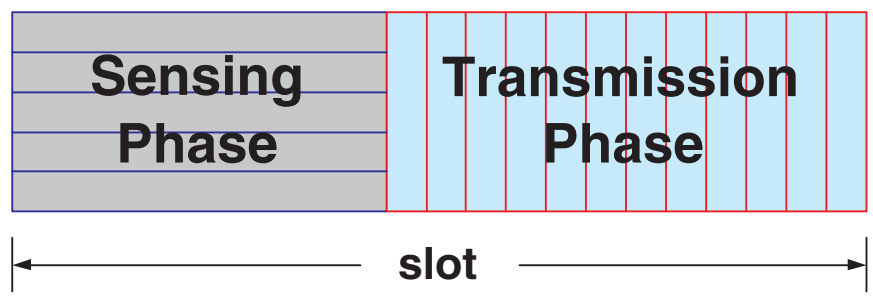

Fig. 1. Slot structure of the secondary network.

The rest of this paper is organized as follows. Section II introduces the system model and formulates an optimization problem of finding the target channel sequence with the objective of minimizing the cumulative handoff delay for a secondary users' connection. Then, the closed-form expression for the cumulative handoff delay is derived in Section III. Furthermore, Section IV presents a dynamic programming algorithm to solve the target channel sequence search problem. Moreover, a suboptimal low-complexity greedy algorithm is suggested in Section V. Numerical results are shown in Section VI. Finally, we give our concluding remarks in Section VII.

\section{Problem Formulation}

\section{A. System Model}

The CR network is considered as a time-slotted system, where the slot of secondary network consists of sensing phase and transmission phase as shown in Fig. 1. Each secondary user must perform spectrum sensing at the beginning of each time slot to detect the presence of primary users ${ }^{2}$. If the current operating channel is assessed as idle, the secondary user can transmit or receive data in the remaining duration of this time slot. Otherwise, the unfinished transmission will be resumed at the preselected target channel by executing spectrum handoff procedures. The kind of listen-before-talk channel access scheme has been implemented in many wireless techniques, such as the quiet period of the IEEE 802.22 standard [22].

Some assumptions are adopted in our performance model for spectrum handoff. First, it is assumed that the primary users' connections have higher priority to access channel than the secondary users' connections. Secondly, the channel access order is scheduled according to the first-come-first-served (FCFS) discipline for the connections with the same priority ${ }^{3}$. Furthermore, each primary and secondary user is pre-assigned with a default channel. After the initial handshaking, the transmitter starts sending data at the default channel to its intended receiver [14]. Lastly, we assume that only one user can transmit data in one channel at any time.

\footnotetext{
${ }^{2}$ Based on this method, the transmission of secondary users may still interfere with the transmission of the primary users due to missed detection. Missed detection occurs when the detector mistakenly reports the absence of a primary user. In this case, data transmission of the primary users will be stained by the secondary users, and then primary users must retransmit the stained data in the next slot. Thus, primary users' actual transmission time is extended as discussed in [21].

${ }^{3}$ In order to apply the first-come-first-served discipline, one network controller may be included in CR networks. For example, the base station of the secondary network can be the network controller. It can record the arrival time of all secondary users' data requests. Next, when channel becomes idle, the base station can notify the suitable secondary users through its control channel in order to initialize data transmission.
} 


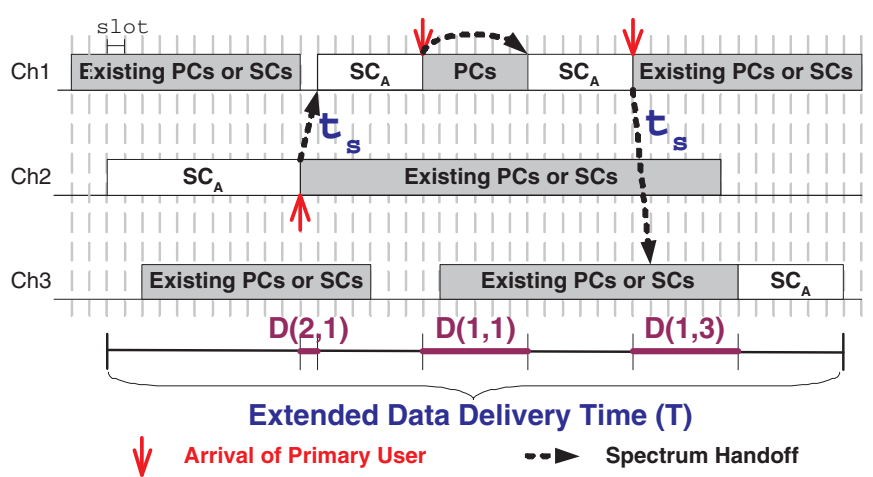

Fig. 2. An example of transmission process for the secondary users' connection $\mathrm{SC}_{A}$, where $t_{S}$ is the channel switching time and $T$ is the extended data delivery time of $\mathrm{SC}_{A}$. The gray areas indicate that the channels are occupied by the existing primary users' connections (PCs) or secondary users' connections (SCs). Because $\mathrm{SC}_{A}$ is interrupted three times in total, the overall data connection is divided into four segments. Note that $\mathbf{E}[D(2,1) \mid \mathrm{Ch} 1$ is idle $]=t_{s}, \mathbf{E}[D(1,1)]=\mathbf{E}\left[Y_{p}^{(1)}\right]$, and $\mathbf{E}[D(1,3) \mid \mathrm{Ch} 3$ is busy $]=t_{s}+\mathbf{E}\left[W_{s}^{(3)} \mid \mathrm{Ch} 3\right.$ is busy $]$.

\section{B. An Illustrative Example for Multiple Spectrum Handoffs}

A secondary users' connection may encounter multiple spectrum handoffs due to more than one interruption from the primary users. Because these spectrum handoffs can be requested at different channels, the secondary users need to select a set of target channels (i.e., the target channel sequence) for a series of spectrum handoffs.

To begin with, we give an example to illustrate the multiple handoff scenario. Note that we use capital letters to denote random variables and lower-case letters to denote specific values of these variables in this paper. Fig. 2 shows that a secondary users' connection $\mathrm{SC}_{A}$ experiences three interruptions from primary users. We assume that the transmitter of $\mathrm{SC}_{A}$ intends to transmit data in a connection using 29 slots in a slotted system. Let random variable $T$ be $\mathrm{SC}_{A}$ 's extended data delivery time, which is the duration from the beginning of sending data until the instant of finishing transmitting the whole data in the originally established 29-slot data connection. Furthermore, denote random variable $D\left(k, k^{\prime}\right)$ as the handoff delay, which is the duration from the instant when the transmission of the secondary user is interrupted at channel $k$ until it is resumed at channel $k^{\prime}$. Then, the transmission process with multiple handoffs is described as follows:

1) In the beginning, $\mathrm{SC}_{A}$ is established at its default channel Ch2. Let $\mathrm{SC}_{A}$ 's predetermined target channel sequence for multiple handoffs be (Ch1, Ch1, Ch3, . ) .

2) At the first interruption, the operating channel of $\mathrm{SC}_{A}$ is changed from $\mathrm{Ch} 2$ to the idle $\mathrm{Ch} 1$ because the first element in the target channel sequence is Ch1. In this case, $\mathbf{E}[D(2,1) \mid \mathrm{Ch} 1$ is idle $]=t_{s}$, where $t_{s}$ is the channel switching time and $\mathbf{E}[\cdot]$ is the expectation function.

3) At the second interruption, $\mathrm{SC}_{A}$ stays on its current operating channel $\mathrm{Ch} 1$ because the second element in the target channel sequence is Ch1. Thus, $\mathrm{SC}_{A}$ cannot be resumed until all the Ch1's high-priority primary users' connections finish their transmissions. Denote the busy period $Y_{p}^{(k)}$ as the busy duration of primary users' connections at $\mathrm{Ch} k$. Then, $\mathbf{E}[D(1,1)]=\mathbf{E}\left[Y_{p}^{(1)}\right]$ in this case.

4) At the third interruption, according to the third element in the target channel sequence, $\mathrm{SC}_{A}$ changes its operating channel to Ch3. However, because Ch3 is busy, $\mathrm{SC}_{A}$ must wait until all the current connections at $\mathrm{Ch} 3$ are served ${ }^{4}$. Denote the waiting time $W_{s}^{\left(k^{\prime}\right)}$ as the duration from the time instant that a secondary connection arrives at channel $k^{\prime}$ until it can start transmitting data at channel $k^{\prime}$. In this case, $\mathbf{E}[D(1,3) \mid \mathrm{Ch} 3$ is busy $]=$ $t_{s}+\mathbf{E}\left[W_{s}^{(3)} \mid \mathrm{Ch} 3\right.$ is busy].

5) Finally, $\mathrm{SC}_{A}$ is completed on $\mathrm{Ch} 3$.

To summarize, the conditional expected handoff delay equals to $\mathbf{E}\left[Y_{p}^{\left(k^{\prime}\right)}\right]$ in the case of $k=k^{\prime}$, where $k$ and $k^{\prime}$ are the operating channels before and after interruption. When $k \neq k^{\prime}$ and channel $k^{\prime}$ is idle, the conditional expected handoff delay equals to $t_{s}+\mathbf{E}\left[W_{s}^{\left(k^{\prime}\right)} \mid\right.$ channel $k^{\prime}$ is idle $]=t_{s}$. However, when $k \neq k^{\prime}$ and channel $k^{\prime}$ is busy, the conditional expected handoff delay equals to $t_{s}+\mathbf{E}\left[W_{s}^{\left(k^{\prime}\right)} \mid\right.$ channel $k^{\prime}$ is busy]. Formally, we have the following three relationships:

$$
\begin{gathered}
\mathbf{E}\left[D\left(k, k^{\prime}\right) \mid k=k^{\prime}\right]=\mathbf{E}\left[Y_{p}^{\left(k^{\prime}\right)}\right], \\
\mathbf{E}\left[D\left(k, k^{\prime}\right) \mid k \neq k^{\prime} \text {, channel } k^{\prime} \text { is idle }\right]=t_{s},
\end{gathered}
$$

and

$$
\begin{aligned}
& \mathbf{E}\left[D\left(k, k^{\prime}\right) \mid k \neq k^{\prime}, \text { channel } k^{\prime} \text { is busy }\right] \\
= & t_{s}+\mathbf{E}\left[W_{s}^{\left(k^{\prime}\right)} \mid \text { channel } k^{\prime} \text { is busy }\right] .
\end{aligned}
$$

\section{Cumulative Handoff Delay Minimization Problem}

As shown in Fig. 2, the cumulative handoff delay from multiple handoffs and the transmission service time contribute to the extended data delivery time. From the viewpoint of maintaining the link connection quality, the extended data delivery time is an important QoS performance metric. Hence, it is important for the secondary users to reduce the cumulative handoff delay from the multiple interruptions of the primary users. Since the cumulative handoff delay is affected by the selected target channels, the target channel sequence design for spectrum handoffs with an objective of minimizing the cumulative handoff delay becomes important QoS issue in CR networks.

Hence, we formulate a Cumulative Handoff Delay Minimization Problem for a link connection with multiple spectrum handoffs. Consider a $\mathrm{CR}$ network $\mathcal{G}$ with $M$ independent channels, where the target channel sequence for future spectrum handoffs is proactively determined for each newly arriving secondary connection. For the secondary connection with the initial default channel $\eta$, we denote its target channel sequence for spectrum handoffs as $\boldsymbol{s}(\eta) \triangleq\left(s_{1}, s_{2}, s_{3}, \cdots\right)$, where $s_{i}$ is the target channel at the $i^{\text {th }}$ interruption. Note that

\footnotetext{
${ }^{4}$ In this paper, we assume that the interrupted secondary user must stay on the selected target channel even though the selected channel is busy and then the interrupted secondary user can transmit the unfinished data only if channel becomes idle. When selecting a busy channel in the current time slot, another approach is to reselect a new channel at the next time slot. However, reselecting a new channel will result in many channel-switching behaviors during a secondary connection. The time complexity of finding the optimal target channel sequence for this approach is very high as discussed in [14].
} 
$s_{0} \triangleq \eta$. Given a set of candidate channels $\Omega=\{1,2, \ldots, M\}$ and the required length of the target channel sequence for $L$ spectrum handoffs, the cumulative handoff delay minimization problem is to determine an optimal target channel sequence (denoted by $s(\eta)^{*}$ ) for a newly arriving secondary users' connection with the minimal average cumulative handoff delay $\mathbf{E}\left[D^{\prime}(\boldsymbol{s}(\eta))\right]$. Formally, we have

$$
\boldsymbol{s}(\eta)^{*}=\underset{\boldsymbol{s}(\eta) \in \Omega^{L}}{\arg \min } \mathbf{E}\left[D^{\prime}(\boldsymbol{s}(\eta))\right]
$$

where $\Omega^{L}$ is the set which consists of all possible target channel sequences with length $L$. In general, this finite value $L$ can be also interpreted as the maximum allowable number of interruptions for a secondary connection. That is, it is assumed that a secondary connection will be dropped if it encounters more than $L$ interruptions.

When the newly arriving secondary connection's optimal target channel sequence is determined, it is unnecessary to re-calculate the optimal target channel sequences of the other existing secondary users' connections in $\mathcal{G}$ because we assume that the new secondary connection has highest priority to determine its optimal target channel sequence. In CR networks, in order to achieve the fairness requirement, it is desired to reduce the variance of the extended data delivery time of all secondary connections. Because the existing secondary connections have initiated their transmission awhile, the remaining transmission time of the existing secondary connections will be shorter than the service time of the newly arriving secondary connection on average. Hence, reducing the extended data delivery time of a new secondary connection is more critical than reducing that of the existing secondary connections. In this case, after the new secondary connection determines its optimal target channel sequence, the existing secondary connections cannot change their predetermined target channel sequences even though these predetermined target channel sequences may no longer be optimal. Based on this approach, the variance of the extended data delivery time of all secondary connections can be reduced. Note that the considered network is fair for all the secondary users because the probabilities of having a new connection for all secondary users are the same.

\section{Cumulative Handoff Delay Analysis}

In this section, we derive the closed-form expression for the average cumulative handoff delay of target channel sequences $s(\eta)$, taking into account of the effects of various arrival rates and service time distributions of the primary and secondary users. The analytical formulas can facilitate the optimal target channel sequence design, which will be discussed in the next section. To ease notations, we denote $s$ for $s(\eta)$ in the rest of this paper. Let $N$ be the total number of interruptions in the considered connection, where $N \leq L$. According to the total probability principle, it follows that

$$
\begin{aligned}
& \mathbf{E}\left[D^{\prime}(\boldsymbol{s})\right] \\
= & \sum_{n=1}^{L} \operatorname{Pr}\{N=n\} \mathbf{E}\left[D^{\prime}(\boldsymbol{s}) \mid N=n\right] \\
= & \sum_{n=1}^{L}\left[\operatorname{Pr}\{N=n\} \sum_{i=1}^{n} \mathbf{E}\left[D\left(s_{i-1}, s_{i}\right)\right]\right],
\end{aligned}
$$

where $D\left(s_{i-1}, s_{i}\right)$ is the handoff delay when a secondary user changes from channel $s_{i-1}$ to $s_{i}$. Note that we use the capital letters (such as $N$ and $D\left(s_{i-1}, s_{i}\right)$ ) to denote random variables, and the lower case letters (such as $n$ and $d\left(s_{i-1}, s_{i}\right)$ ) to denote the corresponding values.

\section{A. Derivation of $\boldsymbol{E}\left[D\left(s_{i-1}, s_{i}\right)\right]$}

Firstly, we evaluate $\mathbf{E}\left[D\left(s_{i-1}, s_{i}\right)\right]$ in (5). When a primary users' connection accesses the channel that is occupied by a secondary user, an interruption event happens to the considered secondary users' connection. Spectrum handoff delay depends on the selected target channel. The interrupted secondary users can either change to another channel or wait for the current channel to become idle again. If the considered secondary users' connection chooses to wait for its current operating channel (i.e., $s_{i-1}=s_{i}$ ), the expected handoff delay is the busy period $Y_{p}^{\left(s_{i}\right)}$, which is defined in Section II. If the considered secondary users' connection changes its operating channel (i.e., $s_{i-1} \neq s_{i}$ ), it cannot be reestablished until all the present traffic workload in the highpriority and low-priority queues of channel $s_{i}$ are served. Let $W_{s}^{\left(s_{i}\right)}$ be the waiting time for the secondary users' connections at channel $s_{i}$. Assume the arrivals of the primary users' connections follow Poisson distribution. Thus, the arrivals of the interrupted secondary users' connections at channel $s_{i}$ also follow Poisson distribution. Recall Poisson arrivals see time average (PASTA) property [23] and apply it to the arrivals of the interrupted secondary users' connections at channel $s_{i}$. We can expect that all the interrupted secondary users' connections at channel $s_{i}$ wait the same time $\mathbf{E}\left[W_{s}^{\left(s_{i}\right)}\right]$ on average until channel $s_{i}$ becomes idle, where the waiting time is uncorrelated with the number of interruptions. Thus, in the case $s_{i-1} \neq s_{i}$, the expected handoff delay is the sum of $\mathbf{E}\left[W_{s}^{\left(s_{i}\right)}\right]$ and the channel switching time $t_{s}$. Hence, it follows that

$$
\mathbf{E}\left[D\left(s_{i-1}, s_{i}\right)\right]=\left\{\begin{array}{lll}
\mathbf{E}\left[Y_{p}^{\left(s_{i}\right)}\right] & , & s_{i-1}=s_{i} \\
\mathbf{E}\left[W_{s}^{\left(s_{i}\right)}\right]+t_{s} & , & s_{i-1} \neq s_{i}
\end{array} .\right.
$$

Note that (6) is equivalent to (1), (2), and (3).

In this paper, we apply the preemptive resume priority (PRP) M/G/1 queueing model, as shown in Fig. 3, to obtain the closed-form expressions for $\mathbf{E}\left[Y_{p}^{\left(s_{i}\right)}\right]$ and $\mathbf{E}\left[W_{s}^{\left(s_{i}\right)}\right]$. Let $\lambda_{p}^{(k)}$ (arrivals/slot) and $\lambda_{s}^{(k)}$ (arrivals/slot) be the initial arrival rates of the primary users' and secondary users' connections at channel $k$ in $\mathcal{G}$, respectively. Represent $X_{p}^{(k)}$ (slots/arrival) and $X_{s}^{(k)}$ (slots/arrival) as the random variables of the corresponding service time of the primary and secondary users, respectively ${ }^{5}$. Note that the traffic loads of the primary and

\footnotetext{
${ }^{5}$ The probability density functions of $\lambda_{p}^{(k)}, \lambda_{s}^{(k)}, X_{p}^{(k)}$, and $X_{s}^{(k)}$ can be obtained by certain traffic pattern prediction methods such as [24].
} 


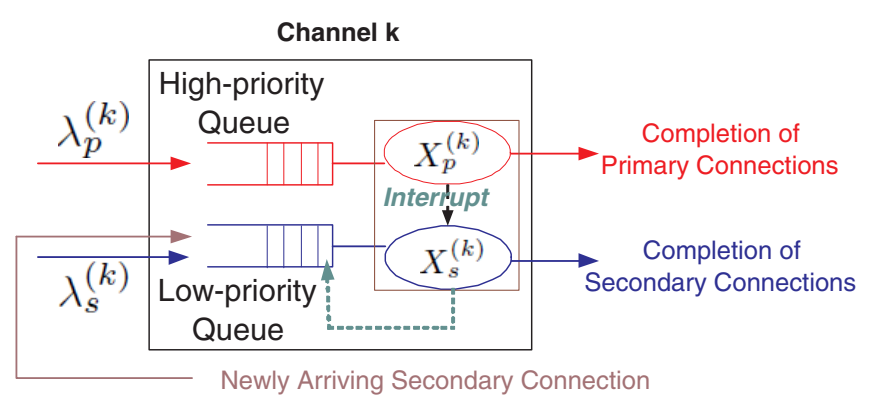

Fig. 3. Preemptive resume priority (PRP) M/G/1 queueing model.

secondary users must enter the high-priority and low-priority queues before transmitting data, respectively. Furthermore, the primary user can interrupt the transmission of the secondary connections.

Next, $\mathbf{E}\left[Y_{p}^{\left(s_{i}\right)}\right]$ and $\mathbf{E}\left[W_{s}^{\left(s_{i}\right)}\right]$ are derived based on the following assumptions. For the newly arriving secondary connection, it can change to new channel or stay on the current operating channel when it is interrupted. In contrast, for the other existing secondary users' connections in $\mathcal{G}$, we assume that their optimal target channel sequences are to always stay on the same channel. That is, they will not change their operating channel for the interruption requests. In this case, the unfinished workload of the interrupted secondary connection will be connected to the head of the low-priority queue of its current operating channel. Under the above assumptions and referring to [25], [26], we obtain the closed-form expressions for $\mathbf{E}\left[Y_{p}^{\left(s_{i}\right)}\right]$ and $\mathbf{E}\left[W_{s}^{\left(s_{i}\right)}\right]$ as follows:

$$
\mathbf{E}\left[Y_{p}^{\left(s_{i}\right)}\right]=\frac{\mathbf{E}\left[X_{p}^{\left(s_{i}\right)}\right]}{1-\lambda_{p}^{\left(s_{i}\right)} \mathbf{E}\left[X_{p}^{\left(s_{i}\right)}\right]},
$$

and

$$
\begin{aligned}
\mathbf{E}\left[W_{s}^{\left(s_{i}\right)}\right]= & \frac{1}{1-\lambda_{p}^{\left(s_{i}\right)} \mathbf{E}\left[X_{p}^{\left(s_{i}\right)}\right]} \times \\
& \frac{\lambda_{p}^{\left(s_{i}\right)} \mathbf{E}\left[X_{p}^{\left(s_{i}\right)}\right]^{2}+\lambda_{s}^{\left(s_{i}\right)} \mathbf{E}\left[X_{s}^{\left(s_{i}\right)}\right]^{2}}{1-\lambda_{p}^{\left(s_{i}\right)} \mathbf{E}\left[X_{p}^{\left(s_{i}\right)}\right]-\lambda_{s}^{\left(s_{i}\right)} \mathbf{E}\left[X_{s}^{\left(s_{i}\right)}\right]} .
\end{aligned}
$$

Substituting (7) and (8) into (6), the closed-form expression for $\mathbf{E}\left[D\left(s_{i-1}, s_{i}\right)\right]$ can be obtained. Although it is assumed that the other existing secondary users' connections in $\mathcal{G}$ will not change its operating channel when interruption occurs, the proposed analytical framework can be also applied to the case that the operating channels of the existing secondary connections are different in spectrum handoffs. The closedform expressions of (7) and (8) for the more general target channel sequences were investigated in [27].

\section{B. Derivation of $\boldsymbol{P r}\{N=n\}$}

Next, we derive the closed-form expression for $\operatorname{Pr}\{N=n\}$ in (5). It follows that

$$
\operatorname{Pr}\{N=n\}=\operatorname{Pr}\{N=n \mid N \geq n\} \operatorname{Pr}\{N \geq n\} .
$$

First, under the condition of having $i$ interruptions already, the probability that a secondary users' connection is interrupted at channel $s_{i}$ again is denoted by $p_{i}^{\left(s_{i}\right)}$. Since $p_{i}^{\left(s_{i}\right)}=\operatorname{Pr}\{N>$ $i \mid N \geq i\}$, we have

$$
\begin{aligned}
& 1-p_{n}^{\left(s_{n}\right)} \\
= & \operatorname{Pr}\{N \geq n \mid N \geq n\}-\operatorname{Pr}\{N>n \mid N \geq n\} \\
= & \operatorname{Pr}\{N=n \mid N \geq n\} .
\end{aligned}
$$

Next it follows that

$$
\begin{aligned}
\operatorname{Pr}\{N \geq n\} & =\prod_{i=0}^{n-1} \frac{\operatorname{Pr}\{N \geq i+1\}}{\operatorname{Pr}\{N \geq i\}} \\
& =\prod_{i=0}^{n-1} \operatorname{Pr}\{N \geq i+1 \mid N \geq i\} \\
& =\prod_{i=0}^{n-1} p_{i}^{\left(s_{i}\right)}
\end{aligned}
$$

because $\operatorname{Pr}\{N \geq 0\}=1$ and $\operatorname{Pr}\{N \geq i+1 \mid N \geq i\}=\frac{\operatorname{Pr}\{N \geq i+1\}}{\operatorname{Pr}\{N \geq i\}} \quad$ Finally, substituting (10) and (11) into (9), one can express the probability that the secondary connection is interrupted exactly $n$ times as follows:

$$
\operatorname{Pr}\{N=n\}=\left(1-p_{n}^{\left(s_{n}\right)}\right) \prod_{i=0}^{n-1} p_{i}^{\left(s_{i}\right)} .
$$

Note that the closed-form expression for $p_{i}^{\left(s_{i}\right)}$ was derived in [27] as follows:

$$
p_{i}^{\left(s_{i}\right)}=\lambda_{p}^{\left(s_{i}\right)} \mathbf{E}\left[\Phi_{i}^{\left(s_{i}\right)}\right],
$$

where the random variable $\Phi_{i}^{(k)}$ is defined to be the transmission duration of the newly arriving secondary connection at channel $k$ between the $i^{t h}$ and the $(i+1)^{t h}$ interruptions.

\section{Optimal Dynamical Programming Algorithm}

In this section, we use the dynamic programming (DP) optimization technique to solve the Cumulative Handoff Delay Minimization Problem. If this problem is solved by exhaustively searching all the possible target channel sequences, this brute force approach must enumerate all the $M^{L}$ possible permutations of the target channel sequences and compute the cumulative handoff delay for each permutation of a target channel sequence. The exhaustive search method has the time complexity of $O\left(M^{L}\right)$ and is infeasible especially for a large value of $M$ or $L$. Hence, it is critical to design a low-complexity optimization algorithm for this problem. To this end, we first develop a state diagram to characterize the evolution of target channel sequences and their corresponding cumulative handoff delay. We observe that the considered optimization problem has the optimal substructure property. Therefore, a dynamic programming algorithm with a time complexity of $O\left(M^{2} L\right)$ is proposed.

\section{A. State Diagram for Target Channel Sequences}

Fig. 4 shows the proposed two-dimensional state diagram in which the state variable $(k, i)$ represents that channel $k$ is selected to be the element in the target channel sequence at the $i^{t h}$ interruption. The initial state of this state diagram model 


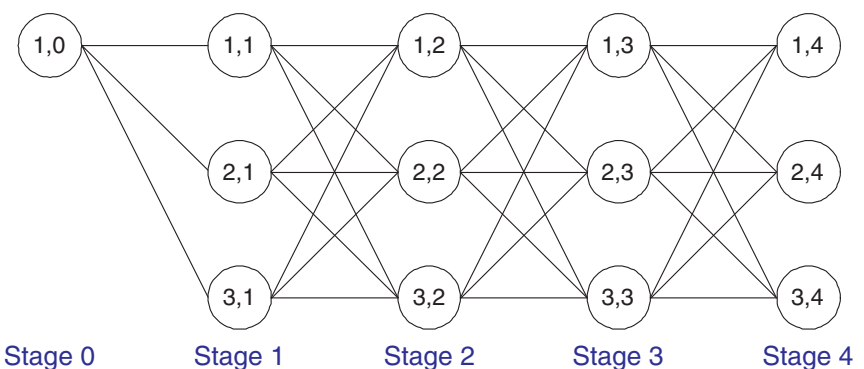

Fig. 4. An example of state diagram of the target channel sequences for a newly arriving secondary connection, where the default channel $\eta=1$, the total number of channels $M=3$, and the required length of the target channel sequence $L=4$. Furthermore, $(k, i)$ stands for the state of operating at the channel $k$ with the $i^{t h}$ interruption.

is $(\eta, 0)$ for the default channel $\eta$. In this example, $\eta=1$, $M=3$, and $L=4$. Stage $i$ represents the set of all possible states at the $i^{t h}$ interruption. The state transitions occur only at the states between the adjacent stages. Specifically, a transition link from $(k, i)$ to $\left(k^{\prime}, i^{\prime}\right)$ exists if $i^{\prime}=i+1$, and vice versa.

In our design, we make the handoff delay of the interrupted secondary users' connection be proportional to the cost of state transition. For example, when the considered secondary users' connection stays on the current channel $k$ at the $i^{t h}$ interruption, the transition cost from states $(k, i-1)$ to $(k, i)$ shall be proportional to $\mathbf{E}[D(k, k)]=\mathbf{E}\left[Y_{p}^{(k)}\right]$. If the considered secondary users' connection changes from channel $k$ to $k^{\prime}\left(k \neq k^{\prime}\right)$ at the $i^{t h}$ interruption, the transition cost from states $(k, i-1)$ to $\left(k^{\prime}, i\right)$ shall be proportional to $\mathbf{E}\left[D\left(k, k^{\prime}\right)\right]=\mathbf{E}\left[W_{s}^{\left(k^{\prime}\right)}\right]+t_{s}$. Denote $w\left(s_{i-1} ; s_{i}, i\right)$ as the transition cost from states $\left(s_{i-1}, i-1\right)$ to $\left(s_{i}, i\right)$. Because transition cost is proportional to the handoff delay of the interrupted secondary users' connections, it follows that

$$
w\left(s_{i-1} ; s_{i}, i\right)=\nu_{i} \cdot \mathbf{E}\left[d\left(s_{i-1}, s_{i}\right)\right],
$$

where $\nu_{i}$ is a normalized factor. In Proposition 1, we will discuss how to obtain $\nu_{i}$.

Proposition 1. Given the total number of interruptions $N$ (where $N \leq L$ ) and the interrupted probability $p_{i}^{\left(s_{i}\right)}$ at channel $s_{i}$ after $i$ interruptions, the normalized factor $\nu_{i}$ can be expressed as follows:

$$
\nu_{i}=\boldsymbol{P r}\{N \geq i\}=\prod_{n=0}^{i-1} p_{n}^{\left(s_{n}\right)} .
$$

Proof: Recall that $\mathbf{E}\left[D^{\prime}(\boldsymbol{s})\right]$ is the average cumulative handoff delay of the considered newly arriving secondary connection with the target channel sequence $s . \mathbf{E}\left[D^{\prime}(\boldsymbol{s})\right]$ can be also interpreted as the cumulative cost for the state transition path $\left(s_{0} \rightarrow s_{1} \rightarrow s_{2} \rightarrow \cdots \rightarrow s_{L}\right)$. Hence, it follows that

$$
\begin{aligned}
\mathbf{E}\left[D^{\prime}(\boldsymbol{s})\right] & =\sum_{i=1}^{L} w\left(s_{i-1} ; s_{i}, i\right) \\
& =\sum_{i=1}^{L}\left[\mathbf{E}\left[D\left(s_{i-1}, s_{i}\right) \cdot \nu_{i}\right]\right] .
\end{aligned}
$$

Furthermore, from (5), we have

$$
\begin{aligned}
& \mathbf{E}\left[D^{\prime}(\boldsymbol{s})\right] \\
= & \sum_{i=1}^{L}\left[\mathbf{E}\left[D\left(s_{i-1}, s_{i}\right)\right] \cdot \operatorname{Pr}\{N \geq i\}\right] \\
= & \sum_{i=1}^{L}\left[\mathbf{E}\left[D\left(s_{i-1}, s_{i}\right)\right] \cdot \prod_{n=0}^{i-1} \operatorname{Pr}\{N \geq n+1 \mid N \geq n\}\right] \\
= & \sum_{i=1}^{L}\left[\mathbf{E}\left[D\left(s_{i-1}, s_{i}\right)\right] \cdot \prod_{n=0}^{i-1} p_{n}^{\left(s_{n}\right)}\right] .
\end{aligned}
$$

Comparing (16) and (17), we prove that (15) is sustained.

\section{B. Optimal Substructure Property}

Next, we show that this considered problem with the proposed state diagram has the optimal substructure property. This problem has the optimal substructure beacuse an optimal solution can be efficiently builded from the optimal solutions to its subproblems [28]. Let $m\left(k^{\prime}, i\right)$ be the cumulative cost of the minimum cost path from the initial state $(\eta, 0)$ to the state $\left(k^{\prime}, i\right)$, where $i \geq 1$. Then, the recursive relationship between $m\left(k^{\prime}, i+1\right)$ and $m\left(k^{\prime}, i\right)$ can be described as follows:

$$
m\left(k^{\prime}, i+1\right)=\min _{k \in \Omega}\left\{m(k, i)+w\left(k ; k^{\prime}, i+1\right)\right\}
$$

where

$$
\begin{aligned}
& m\left(k^{\prime}, 1\right) \\
& =w\left(\eta ; k^{\prime}, 1\right) \\
& =\left\{\begin{array}{lll}
\mathbf{E}\left[Y_{p}^{\left(k^{\prime}\right)}\right] p_{0}^{(\eta)} & , & k^{\prime}=\eta \\
\left(\mathbf{E}\left[W_{s}^{\left(k^{\prime}\right)}\right]+t_{s}\right) p_{0}^{(\eta)} & , & k^{\prime} \neq \eta
\end{array} .\right.
\end{aligned}
$$

Based on this optimal substructure, we can find an optimal solution to the considered optimization problem from the optimal solutions to the subproblems. Consequently, the shortest cumulative handoff delay (denoted by $m^{*}$ ) can be obtained as follows:

$$
m^{*}=\min _{k^{\prime} \in \Omega} m\left(k^{\prime}, L\right) .
$$

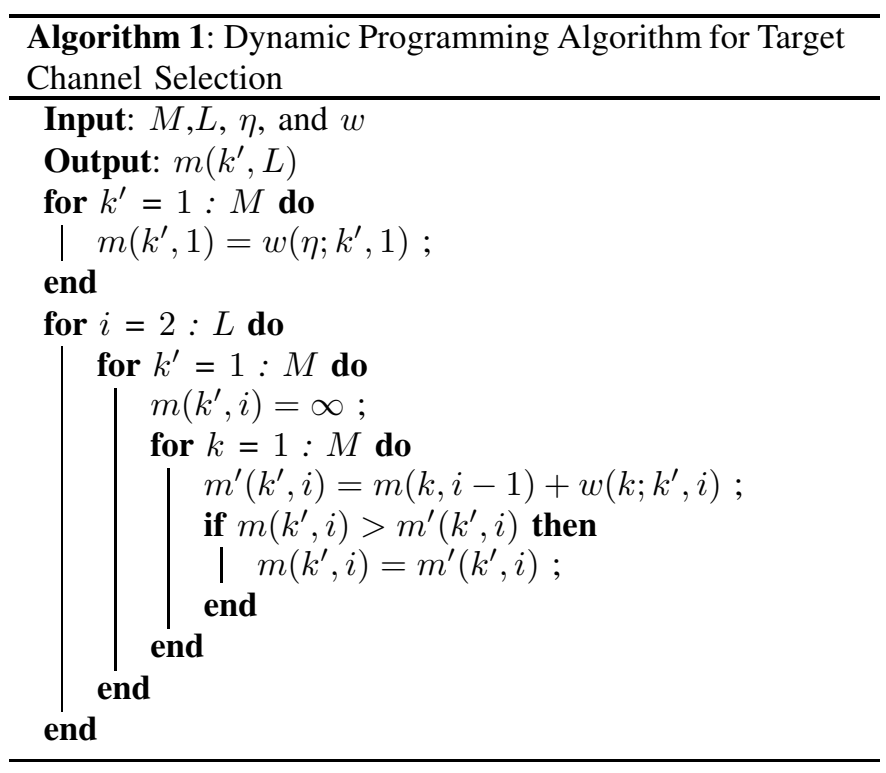




\section{Dynamic-Programming-Based Target Channel Selection Algorithm}

Based on the aforementioned optimal substructure, we propose a DP algorithm with time complexity of $O\left(L M^{2}\right)$ to search the minimum cost path in the state diagram. The objective of the proposed DP algorithm aims at minimizing the cumulative handoff delay of the considered secondary users' connection. Algorithm 1 shows the detailed procedures. When evaluating the cumulative cost $m(k, i)$ at each stage, the selected state variable in the minimum cost path will be recorded. By tracking the selected states in the minimum cost path, the DP algorithm can construct an optimal target channel sequence with the shortest average cumulative handoff delay.

\section{Suboptimal Low-Complexity GReEdy Algorithm}

In the section we present a suboptimal greedy algorithm that can have lower time complexity than the DP-based algorithm for the Cumulative Handoff Delay Minimization Problem. For the DP-based algorithm with time complexity of $O\left(M^{2} L\right)$, the proposed greedy algorithm has time complexity of $O(M)$. Unlike the proposed DP-based algorithm that minimizing the cumulative delay of multiple spectrum handoffs, the greedy strategy for target channel selection only considers the shortest handoff delay of one spectrum handoff.

\section{A. Target Channel Sequences Based on Greedy Strategy}

When the shortest-handoff-delay strategy for target channel sequence design is adopted, some permutations of the target channel sequences in all the $M^{L}$ possible permutations will never occur. Therefore, with a small-sized solution set, the greed strategy can reduce the time complexity in solving the Cumulative Handoff Delay Minimization Problem. Here we give an example to illustrate which permutations of target channel sequence will not be in the solution set if the greedy strategy is adopted. Consider a secondary users' connection whose default channel is channel $1(C h 1)$ in a two-channel system. Either channels 1 or 2 (Ch1 or $C h 2)$ can be the target channel when an interruption event occurs. Assume that the average busy period of $C h 1$ is shorter than the sum of channel switching time and the average waiting time of $C h 2$, i.e., $\mathbf{E}\left[Y_{p}^{(1)}\right]<t_{s}+\mathbf{E}\left[W_{s}^{(2)}\right]$. At the first interruption, the greedy strategy selects $C h 1$ as the target channel for the secondary user. The similar argument can be held for all the upcoming interruptions. Thus, the target channel sequence shall be $(C h 1, C h 1, C h 1, C h 1, C h 1, \cdots)$. As a result, some permutations of the target channel sequences, such as $(C h 1, C h 2, C h 2, C h 2, C h 2, \cdots)$ or

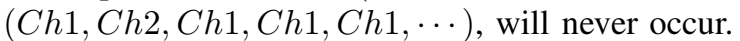

In Theorem 2, even for a general $M$-channel system, we prove that only six target channel sequences are required to be compared when the greedy shortest-handoff-delay target channel selection strategy is employed.

Theorem 2. The shortest-handoff-delay target channel selection strategy only requires comparing six target channel sequences, as shown in Fig. 5.

Proof: Consider a secondary users' connection whose default channel is $\alpha(\alpha \in \Omega)$. The greedy strategy is adopted

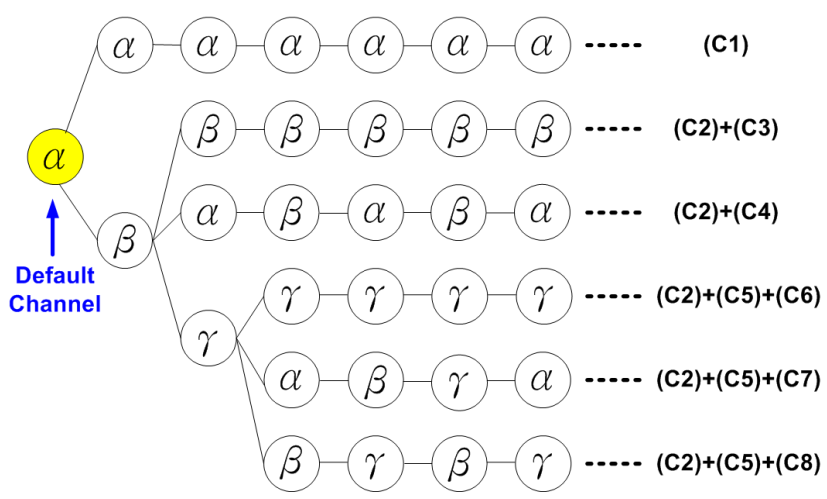

Fig. 5. Six kinds of candidate sequences for the Cumulative Handoff Delay Minimization Problem when the greedy shortest-handoff-delay target channel selection strategy is adopted.

to select a channel with the shortest handoff delay when an interruption event occurs. Thus, the secondary user will compare the expected one-time handoff delay of staying on the same channel and that of changing to a new channel. Next we discuss in which conditions the greedy strategy can exclude some target channel sequences from the solution set.

(1) At the first interruption: The secondary user can select channels $\alpha$ or $k(k \in \Omega /\{\alpha\})$ for the target channel. If staying on channel $\alpha$, the expected delay for the nonhopping spectrum handoff equals the average busy period of the primary users' connections at channel $\alpha$ (denoted by $\left.\mathbf{E}\left[Y_{p}^{(\alpha)}\right]\right)$. If changing to channel $k$, the secondary user will experience the delay of the hopping spectrum handoff, which is the sum of $t_{s}$ and $\mathbf{E}\left[W_{s}^{(k)}\right]$. Hence, if the following condition (C1) is satisfied,

$$
\text { (C1) : } \mathbf{E}\left[Y_{p}^{(\alpha)}\right] \leq \min _{k \in \Omega /\{\alpha\}}\left\{\mathbf{E}\left[W_{s}^{(k)}\right]+t_{s}\right\},
$$

channel $\alpha$ is the first element in the target channel sequence. When the traffic statistics of all channels are stationary and (C1) is satisfied for all the upcoming interruptions, the interrupted secondary user will always stay on channel $\alpha$. That is, the target channel sequence becomes $(\alpha, \alpha, \alpha, \alpha, \alpha, \alpha, \cdots)$, as shown in the first row of Fig. 5.

On the other hand, if the condition (C2) is satisfied,

$$
\text { (C2) : }\left\{\begin{array}{c}
\beta=\underset{k \in \Omega /\{\alpha\}}{\arg \min } \mathbf{E}\left[W_{s}^{(k)}\right] \\
\mathbf{E}\left[W_{s}^{(\beta)}\right]+t_{s}<\mathbf{E}\left[Y_{p}^{(\alpha)}\right]
\end{array},\right.
$$

the first element in the target channel sequence is channel $\beta$. Since (C2) cannot determine the remaining elements in the target channel sequence, we need to discuss the situations at the second interruption.

(2) At the second interruption: Under the condition (C2), the secondary user needs to consider the following three conditions for the target channel at the second interruption: (1) staying on channel $\beta$; (2) switching back to channel $\alpha$; and (3) changing to channel $\gamma(\gamma \neq \alpha$ and $\gamma \neq \beta)$.

- First, we consider the case of staying on channel $\beta$. In this case, the following condition shall be satisfied:

$$
\text { (C3) : } \mathbf{E}\left[Y_{p}^{(\beta)}\right] \leq \min _{k \in \Omega /\{\beta\}}\left\{\mathbf{E}\left[W_{s}^{(k)}\right]+t_{s}\right\}
$$


When (C3) is satisfied, the interrupted secondary user prefers staying on channel $\beta$ similar to the situation of (C1). Combining (C2) and (C3) leads to the target channel sequence $(\beta, \beta, \beta, \cdots)$, as shown in the second row of Fig. 5.

- Next, we consider the case of switching back to channel $\alpha$. In this case, it follows that

$$
\begin{aligned}
\text { (C4) : } & \mathbf{E}\left[W_{s}^{(\alpha)}\right]+t_{s}< \\
& \min \left\{\min _{k \in \Omega /\{\alpha, \beta\}}\left\{\mathbf{E}\left[W_{s}^{(k)}\right]+t_{s}\right\}, \mathbf{E}\left[Y_{p}^{(\beta)}\right]\right\} .
\end{aligned}
$$

With (C4), the interrupted secondary user will switch back to channel $\alpha$. If the third interruption event occurs, then the secondary user will switch back to channel $\beta$ according to (C2). Hence, combining (C2) and (C4) yields the target channel sequence $(\beta, \alpha, \beta, \alpha, \beta, \alpha, \cdots)$, as shown in the third row of Fig. 5.

- When (C3) and (C4) are not satisfied, the case of changing to channel $\gamma$ is considered. It implies that there exists channel $\gamma(\gamma \neq \alpha)$ such that

$$
\text { (C5) : }\left\{\begin{array}{l}
\gamma=\underset{k \in \Omega /\{\alpha, \beta\}}{\arg \min } \mathbf{E}\left[W_{s}^{(k)}\right] \\
\mathbf{E}\left[W_{s}^{(\gamma)}\right]+t_{s}<\mathbf{E}\left[Y_{p}^{(\beta)}\right] \\
\mathbf{E}\left[W_{s}^{(\gamma)}\right]<\mathbf{E}\left[W_{s}^{(\alpha)}\right]
\end{array} .\right.
$$

Although the second element in the target channel sequence is channel $\gamma$, combining (C2) and (C5) cannot determine the remaining elements in the target channel sequence. We need to further discuss the situations at the third interruption.

(3) At the third interruption: Given (C2) and (C5), three more situations are needed to be considered as follows: (1) staying on channel $\gamma$; (2) switching back to channel $\alpha$; and (3) switching back to channel $\beta$.

- First of all, the interrupted secondary user prefers staying on channel $\gamma$ if the following condition is sustained.

$$
\text { (C6) : } \mathbf{E}\left[Y_{p}^{(\gamma)}\right] \leq \min _{k \in \Omega /\{\gamma\}}\left\{\mathbf{E}\left[W_{s}^{(k)}\right]+t_{s}\right\}
$$

Specifically, combining (C2), (C5), and (C6) results in the target channel sequence $(\beta, \gamma, \gamma, \gamma, \cdots)$, as shown in the fourth row of Fig. 5.

- The interrupted secondary user will select channel $\alpha$ if the following condition is satisfied:

$$
\begin{aligned}
(\mathbf{C} 7): \mathbf{E}\left[W_{s}^{(\alpha)}\right]+t_{s}< & \\
& \min \left\{\min _{k \in \Omega /\{\alpha, \gamma\}}\left\{\mathbf{E}\left[W_{s}^{(k)}\right]+t_{s}\right\}, \mathbf{E}\left[Y_{p}^{(\gamma)}\right]\right\} .
\end{aligned}
$$

Because condition (C2) is satisfied in this condition, the target channel at the fourth interruption will be channel $\beta$. Consequently, condition (C5) will make that channel $\gamma$ becomes the target channel at the fifth interruption. In this case, condition (C7) will be checked again. Thus, when (C2), (C5) and (C7) are satisfied, the target channel sequence becomes $(\beta, \gamma, \alpha, \beta, \gamma, \alpha, \beta, \gamma, \alpha, \cdots)$, as shown in the fifth row of Fig. 5 .

- The target channel at the third interruption is channel $\beta$ if the following condition is satisfied:

$$
\text { (C8): } \begin{aligned}
\mathbf{E}\left[W_{s}^{(\beta)}\right]+t_{s}< \\
\quad \min \left\{\min _{k \in \Omega /\{\beta, \gamma\}}\left\{\mathbf{E}\left[W_{s}^{(k)}\right]+t_{s}\right\}, \mathbf{E}\left[Y_{p}^{(\gamma)}\right]\right\} .
\end{aligned}
$$

Similar to the discussion in condition (C7), we can prove that combining conditions (C2), (C5) and (C8), the target channel sequence is $(\beta, \gamma, \beta, \gamma, \beta, \gamma, \cdots)$, as shown in the sixth row of Fig. 5.

- Last, we show that it is unnecessary to consider other channels except for channels $\alpha, \beta$, and $\gamma$ at the third interruption. We will prove this property by contradiction. Assume that there exists channel $\xi=\underset{k \in \Omega /\{\alpha, \beta, \gamma\}}{\arg \min } \mathbf{E}\left[W_{s}^{(k)}\right]$ to be another target channel based on the greedy algorithm. That is, the following conditions are sustained:

$$
\left\{\begin{array}{l}
\mathbf{E}\left[W_{s}^{(\xi)}\right]+t_{s}<\mathbf{E}\left[Y_{p}^{(\gamma)}\right] \\
\mathbf{E}\left[W_{s}^{(\xi)}\right]<\mathbf{E}\left[W_{s}^{(\alpha)}\right] \\
\mathbf{E}\left[W_{s}^{(\xi)}\right]<\mathbf{E}\left[W_{s}^{(\beta)}\right]
\end{array} .\right.
$$

Combining conditions $\xi=\arg \min \mathbf{E}\left[W_{s}^{(k)}\right]$, $\mathbf{E}\left[W_{s}^{(\xi)}\right]<\mathbf{E}\left[W_{s}^{(\alpha)}\right]$, and $\mathbf{E}\left[W_{s}^{(\xi)}\right]<\mathbf{E}\left[W_{s}^{(\beta)}\right]$, it follows that

$$
\mathbf{E}\left[W_{s}^{(\xi)}\right]+t_{s}<\mathbf{E}\left[W_{s}^{(k)}\right]+t_{s}, \quad \forall k \neq \xi, \gamma .
$$

However, based on (C2), we know that

$$
\mathbf{E}\left[W_{s}^{(\beta)}\right]+t_{s}<\mathbf{E}\left[W_{s}^{(k)}\right]+t_{s}, \quad \forall k \neq \alpha, \beta .
$$

Contradiction occurs because (21) yields $\mathbf{E}\left[W_{s}^{(\xi)}\right]<$ $\mathbf{E}\left[W_{s}^{(\beta)}\right]$, whereas (22) yields $\mathbf{E}\left[W_{s}^{(\beta)}\right]<\mathbf{E}\left[W_{s}^{(\xi)}\right]$. Hence, it is proved that it is unnecessary to further consider other channels except for channels $\alpha, \beta$, and $\gamma$.

From the above discussions, conditions (C1)-(C8) are all needed to be examined if the greedy strategy is adopted. We conclude that the greedy strategy results in only six possible target channel sequences that are needed to be further compared in the Cumulative Handoff Delay Minimization Problem, which are shown in Fig. 5.

Based on Theorem 2, the transmitter and the receiver need to consider only three channels for spectrum handoff when the greedy target channel selection strategy is adopted. This small-sized solution set with the greed strategy can simplify channel consensus issue in CR networks. Theorem 2 can be also extended to other greedy strategies based on various criteria, such as the longest expected remaining idle period.

\section{B. Greedy Target Channel Selection Algorithm}

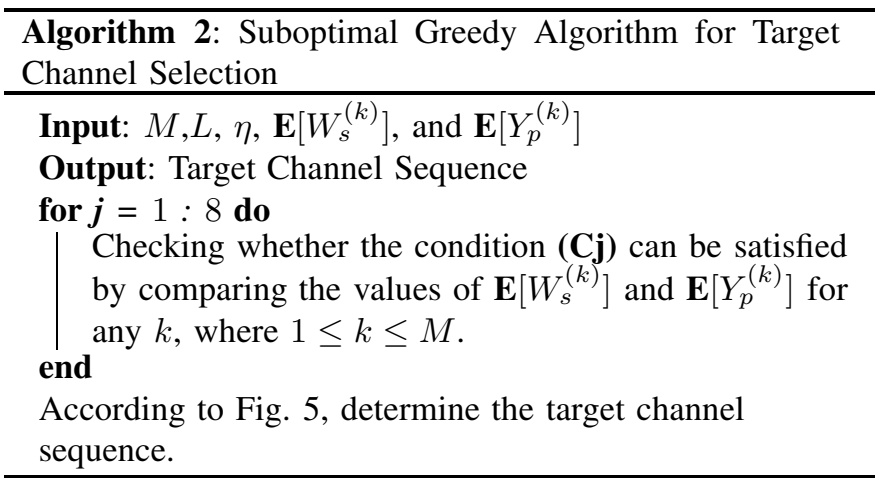


Based on the above discussions, the target channel selection algorithm with $O(M)$ time complexity is proposed as follows. As seen in Algorithm 2, by examining conditions (C1) to (C8) one time, one can determine a suboptimal target channel sequence by referring to Fig. 5 .

\section{Numerical Results}

In this section, we show the performance of cumulative handoff delay for the proposed target channel sequence design approaches in an environment with multiple handoffs. The proposed analytical models can incorporate the effects of various statistics of service time distributions of both primary and secondary users. Five target channel selection schemes are compared, consisting of (1) a random selection strategy; (2) throughput-based strategy; (3) the proposed greedy shortesthandoff-delay target channel selection strategy; (4) the proposed dynamic-programming (DP)-based approach; and (5) the exhaustive-search (ES)-based approach. In our simulations, random selection strategy selects the target channel from candidate channels with equal probability when an interruption event occurs. The throughput-orientated strategy selects the channel that is most unlikely accessed by the primary users. The greedy strategy selects the target channel with the shortest delay in one spectrum handoff. The DP-based and ES-based approaches select the target channel taking into account of the cumulative delay of multiple spectrum handoffs. Nevertheless, the DP-based approach selects the target channel with less search complexity compared to the ES-based approach that requires comparing all the possible permutations of target channel sequences. Note that we consider the case that $\mathrm{L}$ is an enough large value (say 100) in the following numerical results.

To apply the proposed analytical results in (5), we need to specify the effective service time $\Phi_{i}^{\left(s_{i}\right)}$ of the secondary connection between the $i^{\text {th }}$ and the $(i+1)^{\text {th }}$ interruptions in (13). Assume that the service time (denoted by $\chi_{s}$ ) of the considered newly arriving secondary connection is exponentially distributed. Referring to [29], we have $\mathbf{E}\left[\Phi_{i}^{\left(s_{i}\right)}\right]=\frac{\mathbf{E}\left[\chi_{s}\right]}{\lambda_{p}^{\left(s_{i}\right)} \mathbf{E}\left[\chi_{s}\right]+1}$. Thus, it follows that

$$
p_{i}^{\left(s_{i}\right)}=\frac{\lambda_{p}^{\left(s_{i}\right)} \mathbf{E}\left[\chi_{s}\right]}{\lambda_{p}^{\left(s_{i}\right)} \mathbf{E}\left[\chi_{s}\right]+1} .
$$

Note that the distributions of service time $\chi_{s}$ for the newly arriving secondary users' connection and $X_{s}^{(\eta)}$ for the existing secondary users' connections can be different in our model.

\section{A. Effects of Traffic Statistics for Newly Arriving Secondary Connection's Service Time}

Firstly, we investigate the effects of the newly arriving secondary connection's average service time $\left(\mathbf{E}\left[\chi_{s}\right]\right)$ on its cumulative handoff delay. Figure 6 compares the cumulative handoff delay of the five considered target channel selection schemes in a four-channel CR network with $\lambda_{p}^{(k)}=0.02$ and $\lambda_{s}^{(k)}=0.01$ for $1 \leq k \leq 4$. Fig. 6 (a) considers the case where the service time distributions of the primary users in different channels are similar, and Fig. 6 (b) is for the case where the service time distributions of the primary user in different channels are different. From the figure, we have the following observations:

- As the average service time $\mathbf{E}\left[\chi_{s}\right]$ increases, it is expected that a secondary user experiences more interruptions on average. Both figures show the cumulative handoff delay increases as $\mathbf{E}\left[\chi_{s}\right]$ increases.

- Among the five considered target channel selection schemes, the DP-based approach and the exclusive search approach perform the best in both cases. Compared to the random strategy at $\mathbf{E}\left[\chi_{s}\right]=20$, the DP-based approach can reduce the cumulative handoff delay by $20 \%$ and $60 \%$ in Figs. 6(a) and 6(b), respectively. This is because the DP-based approach takes the traffic statistics of both primary users and secondary users into account when determining the target channel for spectrum handoff. The greedy strategy has the same performance as the DPbased approach in Fig. 6(a), but its cumulative spectrum handoff delay is $9 \%$ higher than the DP-based approach at $\mathbf{E}\left[\chi_{s}\right]=20$ in Fig. 6(b).

- It is found that the throughput-based strategy yields the same cumulative handoff delay as the greedy strategy in Fig. 6(b). However, when the statistics of the existing secondary users' connections are different, Fig. 6(a) shows that throughput-based strategy has the worst cumulative handoff delay performance. At $\mathbf{E}\left[\chi_{s}\right]=20$, the cumulative handoff delay of the throughput-orientated strategy is $46 \%$ higher than that of the greedy strategy, and even $16 \%$ worse compared to the random strategy. This can be explained by the fact that the throughputbased target channel selection considers only the traffic statistics of primary users.

\section{B. Effects of Traffic Statistics of the Existing Secondary Con- nections}

Figure 7 shows how the existing secondary connections' traffic statistics (the average service time $\mathbf{E}\left[X_{s}\right]$ and the arrival rate $\lambda_{s}$ ) affect the cumulative handoff delay of the newly arriving secondary connection. Assume that the service time $\chi_{s}$ of the newly arriving secondary users' connection is exponentially distributed with $\mathbf{E}\left[\chi_{s}\right]=10$, and the traffic parameters of the primary users are similar in different channels. Figs. 7(a) and (b) consider the effects of $\mathbf{E}\left[X_{s}\right]$ and the arrival rate $\lambda_{s}$, respectively.

In Fig. 7(a) where $\mathbf{E}\left[X_{s}^{(k)}\right]=\mathbf{E}\left[X_{s}\right]$ for $1 \leq k \leq 4$, we observe the following phenomenons:

- In the range of small $\mathbf{E}\left[X_{s}\right]$ (e.g., $\mathbf{E}\left[X_{s}\right]<15$ ), the cumulative handoff delay increases as $\mathbf{E}\left[X_{s}\right]$ increases for all the target channel selection strategies except for the throughput-based strategy. In our simulation, channel 1 has the lowest busy probability of the primary users. Thus, the throughput-based strategy always selects channel 1 for the target channel, resulting in the constant handoff delay $\mathbf{E}\left[Y_{p}\right]$ independent of $\mathbf{E}\left[X_{s}\right]$.

- Now we discuss the performance comparison in the range of large $\mathbf{E}\left[X_{s}\right]$ (i.e., $\mathbf{E}\left[X_{s}\right] \geq 15$ ). When changing the operating channel, the relation between $\mathbf{E}\left[X_{s}\right]$ and the waiting time of the secondary user is based on (8). Thus, the higher the value of $\mathbf{E}\left[X_{s}\right]$, the longer the waiting time. 


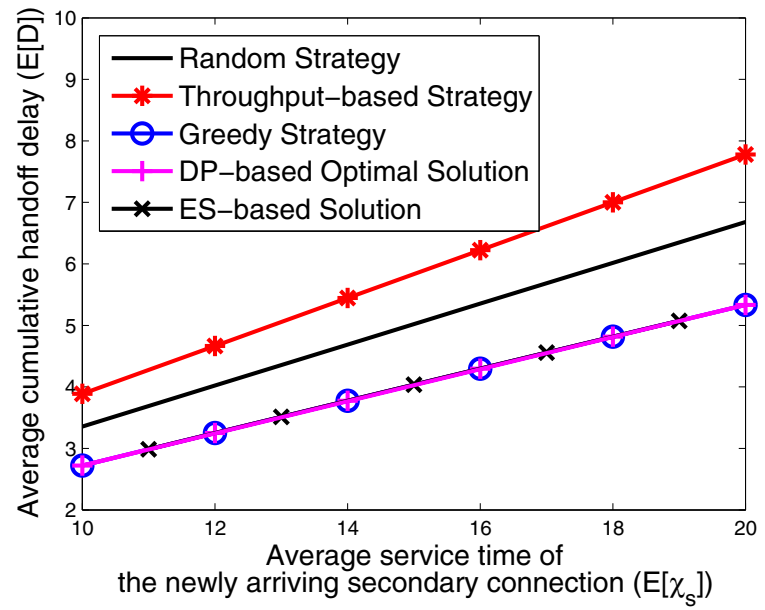

(a)

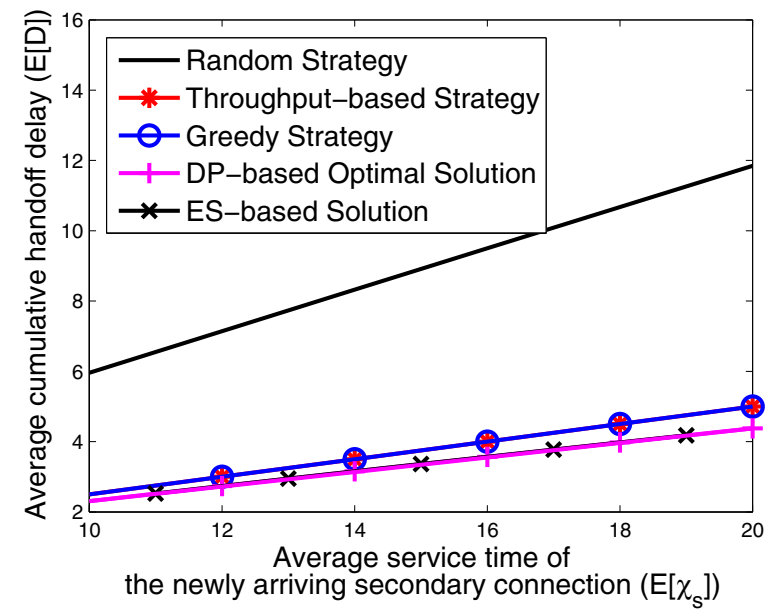

(b)

Fig. 6. Effects of the newly arriving secondary connection's average service time $\mathbf{E}\left[\chi_{s}\right]$ on the cumulative handoff delay for $\lambda_{p}^{(k)}=0.02$ and $\lambda_{s}^{(k)}=0.01$ when $1 \leq k \leq 4$, where (a) $\left(\mathbf{E}\left[X_{p}^{(1)}\right], \mathbf{E}\left[X_{p}^{(2)}\right], \mathbf{E}\left[X_{p}^{(3)}\right], \mathbf{E}\left[X_{p}^{(4)}\right]\right)=(14,15,15,15)$, and $\left(\mathbf{E}\left[X_{s}^{(1)}\right], \mathbf{E}\left[X_{s}^{(2)}\right], \mathbf{E}\left[X_{s}^{(3)}\right], \mathbf{E}\left[X_{s}^{(4)}\right]\right)=(10,12,14,16) ;(b)$ $\left(\mathbf{E}\left[X_{p}^{(1)}\right], \mathbf{E}\left[X_{p}^{(2)}\right], \mathbf{E}\left[X_{p}^{(3)}\right], \mathbf{E}\left[X_{p}^{(4)}\right]\right)=(10,15,20,25)$, and $\left(\mathbf{E}\left[X_{s}^{(1)}\right], \mathbf{E}\left[X_{s}^{(2)}\right], \mathbf{E}\left[X_{s}^{(3)}\right], \mathbf{E}\left[X_{s}^{(4)}\right]\right)=(10,10,10,10)$

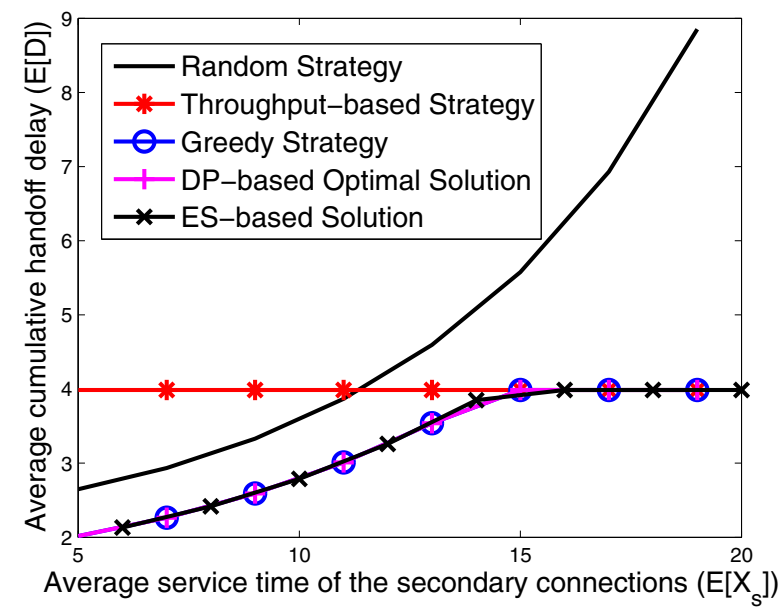

(a) Effect of the average service time $\mathbf{E}\left[X_{s}\right]$ on the cumulative handoff delay $\mathbf{E}[D]$.

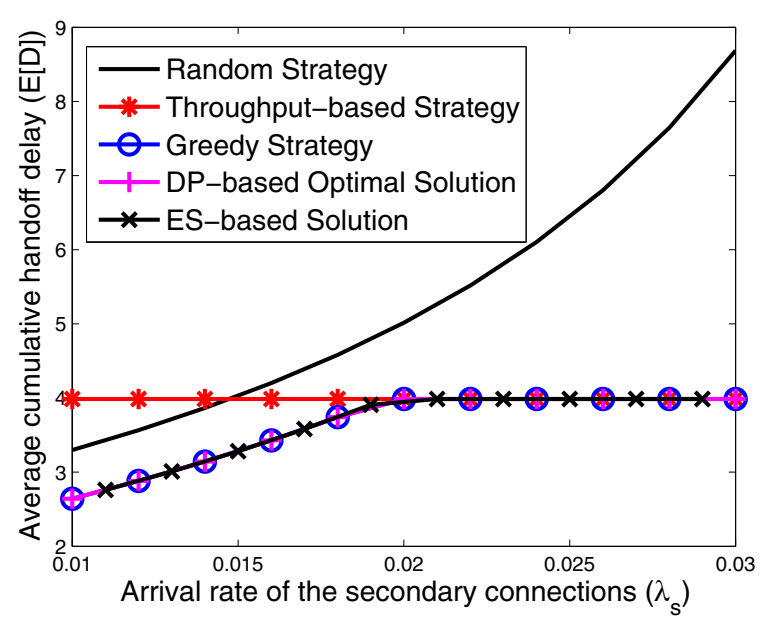

(b) Effect of the arrival rate $\lambda_{s}$ on the cumulative handoff delay $\mathbf{E}[D]$.

Fig. 7. Effect of the average service time $\mathbf{E}\left[X_{s}\right]$ and the arrival rate $\lambda_{s}$ of the secondary users' connections on the cumulative handoff delay of the newly arriving secondary users' connection for $\left(\lambda_{p}^{(1)}, \lambda_{p}^{(2)}, \lambda_{p}^{(3)}, \lambda_{p}^{(4)}\right)=(0.019,0.02,0.02,0.02)$ and $\left(\mathbf{E}\left[X_{p}^{(1)}\right], \mathbf{E}\left[X_{p}^{(2)}\right], \mathbf{E}\left[X_{p}^{(3)}\right], \mathbf{E}\left[X_{p}^{(4)}\right]\right)=(15,15,15,15)$, where (a) $\left(\lambda_{s}^{(1)}, \lambda_{s}^{(2)}, \lambda_{s}^{(3)}, \lambda_{s}^{(4)}\right)=(0.01,0.015,0.02,0.025)$; (b) $\left(\mathbf{E}\left[X_{s}^{(1)}\right], \mathbf{E}\left[X_{s}^{(2)}\right], \mathbf{E}\left[X_{s}^{(3)}\right], \mathbf{E}\left[X_{s}^{(4)}\right]\right)=(10,12,14,16)$.

As a result, for a large value of $\mathbf{E}\left[X_{s}\right]$, the optimal DPbased as well as the ES-based approaches, and the greedy strategy prefer staying on the current operating channel to reduce the impacts of long waiting time due to changing the operating channels, resulting in the same constant handoff delay $\mathbf{E}\left[Y_{p}\right]$. Because the random strategy still possibly change the target channels for spectrum handoff even for a large value of $\mathbf{E}\left[X_{s}\right]$, its cumulative handoff delay increases as $\mathbf{E}\left[X_{s}\right]$ increases as shown in the figure.

Fig. 7(b) shows the effect of $\lambda_{s}$, where $\lambda_{s}^{(k)}=\lambda_{s}$ for $1 \leq k \leq$ 4. As Fig. 7(a) the similar phenomenon can be found except that the saturation point for the cumulative handoff delay is around $\lambda_{s}=0.02$.

\section{Effects of Traffic Statistics of Primary Connections}

Figure 8 shows the effects of the average service time $\mathbf{E}\left[X_{p}\right]$ and the arrival rate $\lambda_{p}$ of the primary users' connections on the cumulative handoff delay of the newly arriving secondary users' connection. Let $\lambda_{s}^{(k)}=\lambda_{s}$ and $\mathbf{E}\left[X_{s}^{(k)}\right]=\mathbf{E}\left[X_{s}\right]$ for $1 \leq k \leq 4$, and the mean of the exponentially distributed service time $\mathbf{E}\left[\chi_{s}\right]=10$.

From Fig. 8(a) where $\mathbf{E}\left[X_{p}^{(k)}\right]=\mathbf{E}\left[X_{p}\right]$ for $1 \leq k \leq 4$, we observe the following phenomenons. As expected, the cumulative handoff delay of all the target channel selection approaches increases as $\mathbf{E}\left[X_{p}\right]$ increases because a larger value of $\mathbf{E}\left[X_{p}\right]$ lead to heavier traffic load. However, the cumulative handoff delay for the random target channel selection strategy increases much more dramatically than the other target chan- 


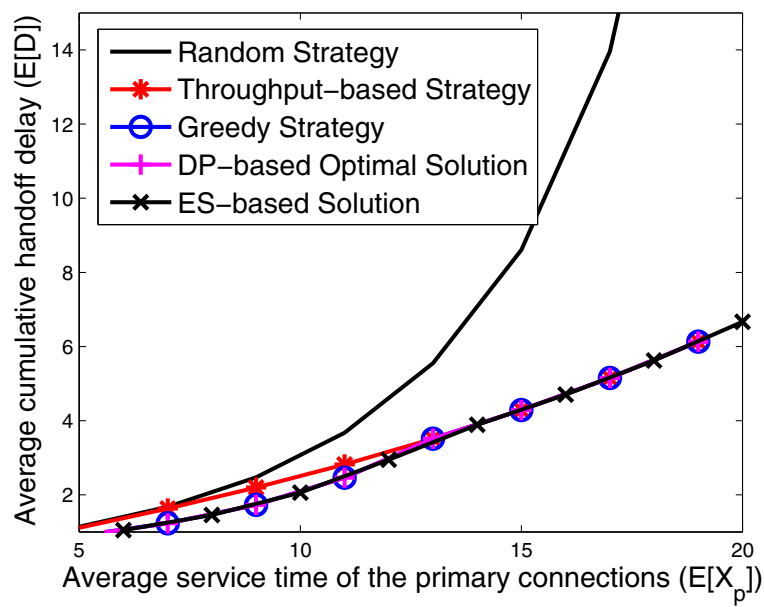

(a) Effect of the average service time $\mathbf{E}\left[X_{p}\right]$ on the cumulative handoff delay $\mathbf{E}[D]$.

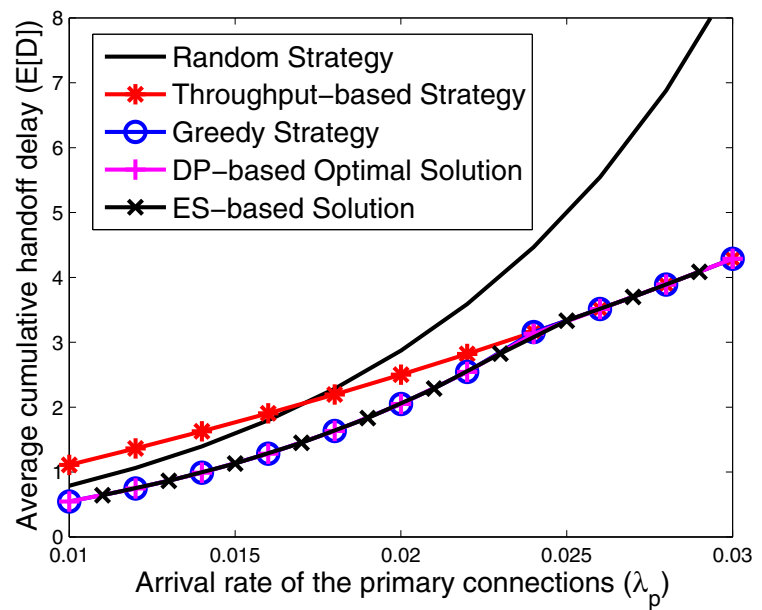

(b) Effect of the arrival rate $\lambda_{p}$ on the cumulative handoff delay $\mathbf{E}[D]$.

Fig. 8. Effect of the average service time $\mathbf{E}\left[X_{p}\right]$ and the arrival rate $\lambda_{p}$ of the primary users' connections on the cumulative handoff delay of the newly arriving secondary connection for $\lambda_{s}^{(k)}=0.01$ and $\mathbf{E}\left[X_{s}^{(k)}\right]=15$ when $1 \leq k \leq 4$, where (a) $\left(\lambda_{p}^{(1)}, \lambda_{p}^{(2)}, \lambda_{p}^{(3)}, \lambda_{p}^{(4)}\right)=(0.02,0.025,0.03,0.035)$; (b) $\left(\mathbf{E}\left[X_{p}^{(1)}\right], \mathbf{E}\left[X_{p}^{(2)}\right], \mathbf{E}\left[X_{p}^{(3)}\right], \mathbf{E}\left[X_{p}^{(4)}\right]\right)=(10,12,14,16)$.

nel selection strategies. This phenomenon can be explained as follows. It is likely that the random selection strategy will still change to other operating channels even in the heavy traffic situation. By contrast, the optimal DP-based as well as ESbased approaches, and the greedy strategy take into account of the traffics of primary users and will wait at the original channels when $\mathbf{E}\left[X_{p}\right] \geq 13$. In this case, their handoff delay is only related to the busy period $\mathbf{E}\left[Y_{p}\right]$, thereby leading to the convergence of the cumulative handoff delay as shown in the region of $\mathbf{E}\left[X_{p}\right] \geq 13$. Fig. 8(b) shows the effect of the primary users' arrival rate $\lambda_{p}$, where $\lambda_{p}^{(k)}=\lambda_{p}$ for $1 \leq k \leq 4$. We can draw the similar conclusion as in Fig. 8(a). In the considered conditions, one can observe that the greedy strategy performs the same as the optimal DP-based and ES-based approaches.

\section{CONCLUSIONS}

In this paper, we have investigated the Cumulative Handoff Delay Minimization Problem for cognitive radio networks. We formulated an optimization problem to determine a minimal-cumulative-handoff-delay target channel sequence when a secondary connection encounters multiple handoffs. We first proposed a trellis state diagram to characterize the cumulative handoff delay cost for various target channel sequences. Next we proposed a dynamic programming algorithm to solve the cumulative handoff delay minimization problem. For a $M$-channel CR network with $L$ spectrum handoffs, the time complexity of the DP-based optimal algorithm is only $O\left(M^{2} L\right)$ compared to $O\left(M^{L}\right)$ of the exclusive search approach. We further suggested a suboptimal but lowcomplexity greedy target channel selection strategy, which has only time complexity of $O(M)$ and requires only six target channel sequences for comparison. Our results show that the performance of this greedy strategy can approach the optimal solution in most cases. However, in the case when the primary users have different service time distributions among the considered channels, the greedy strategy cannot achieve the same performance as the optimal DP-based approach.

Some possible research issues that can be extended from this work include the following. First, it is worthwhile investigating the cumulative handoff delay performance of different target channel selection algorithms in a more general case where the existing secondary users in $\mathcal{G}$ can change their operating channels for the interruption requests. Furthermore, it is still an open research issue to develop a general framework to determine the optimal target channel sequences of all the secondary connections simultaneously.

\section{REFERENCES}

[1] I. F. Akyildiz, W.-Y. Lee, M. C. Vuran, and S. Mohanty, "A survey on spectrum management in cognitive radio networks," IEEE Commun. Mag., vol. 46, no. 4, pp. 40-48, Apr. 2008.

[2] L.-C. Wang, C.-W. Wang, and K.-T. Feng, "A queueing-theoretical framework for QoS-Enhanced spectrum management in cognitive radio networks," IEEE Wireless Commun. Mag., vol. 18, no. 6, pp. 18-26, Dec. 2011.

[3] H. Kim and K. G. Shin, "Efficient discovery of spectrum opportunities with MAC-layer sensing in cognitive radio networks," IEEE Trans. Mobile Comput., vol. 7, no. 5, pp. 533-545, May 2008.

[4] — "Fast discovery of spectrum opportunities in cognitive radio networks," 2008 IEEE International Symp. Dynamic Spectrum Access Netw.

[5] S. Zheng, X. Yang, S. Chen, and C. Lou, "Target channel sequence selection scheme for proactive-decision spectrum handoff," IEEE Commun. Lett., vol. 15, no. 12, pp. 1332-1334, Dec. 2011.

[6] H. Jiang, L. Lai, R. Fan, and H. V. Poor, "Optimal selection of channel sensing order in cognitive radio," IEEE Trans. Wireless Commun., vol. 8, no. 1, pp. 297-307, Jan. 2009.

[7] R. Fan and H. Jiang, "Channel sensing-order setting in cognitive radio networks: a two-user case," IEEE Trans. Veh. Technol., vol. 58, no. 9, pp. 4997-5008, Nov. 2009.

[8] N. B. Chang and M. Liu, "Optimal channel probing and transmission scheduling for opportunistic spectrum access," IEEE/ACM Trans. Netw., vol. 17, no. 6, pp. 1805-1818, Dec. 2009.

[9] C.-W. Wang, L.-C. Wang, and F. Adachi, "Modeling and analysis for reactive-decision spectrum handoff in cognitive radio networks," 2010 IEEE Global Commun. Conf.

[10] C.-W. Wang and L.-C. Wang, "Analysis of reactive spectrum handoff in cognitive radio networks," accepted by IEEE J. Sel. Areas Commun., 2012. 
[11] J. Gambini, O. Simeone, Y. Bar-Ness, U. Spagnolini, and T. Yu, "Packetwise vertical handover for unlicensed multi-standard spectrum access with cognitive radios," IEEE Trans. Wireless Commun., vol. 7, no. 12, pp. 5172-5176, Dec. 2008.

[12] J. Gambini, O. Simeone, U. Spagnolini, Y. Bar-Ness, and Y. Kim, "Cognitive radio with secondary packet-by-packet vertical handover," 2008 IEEE International Conf. Commun.

[13] C.-M. Lee, J.-S. Lin, Y.-P. Hsu, and K.-T. Feng, "Design and analysis of optimal channel-hopping sequence for cognitive radio networks," 2010 IEEE Wireless Commun. Netw. Conf.

[14] Q. Zhao, L. Tong, A. Swami, and Y. Chen, "Decentralized cognitive MAC for opportunistic spectrum access in ad hoc networks: a POMDP framework," IEEE J. Sel. Areas Commun., vol. 25, no. 3, pp. 589-600, Apr. 2007.

[15] Q. Zhao, S. Geirhofer, L. Tong, and B. M. Sadler, "Opportunistic spectrum access via periodic channel sensing," IEEE Trans. Signal Process., vol. 56, no. 2, pp. 785-796, Feb. 2008.

[16] O. Mehanna, A. Sultan, and H. E. Gamal, "Blind cognitive MAC protocols," 2009 IEEE International Conf. Commun.

[17] S. Senthuran, A. Anpalagan, and O. Das, "A predictive opportunistic access scheme for cognitive radios," 2009 IEEE Veh. Technol. Conf. Fall.

[18] A. W. Min and K. G. Shin, "Exploiting multi-channel diversity in spectrum-agile networks," 2008 IEEE International Conf. Comput. Commun.

[19] L. Yang, L. Cao, and H. Zheng, "Proactive channel access in dynamic spectrum networks," Physical Commun., vol. 1, no. 2, pp. 103-111, 2008.

[20] R.-T. Ma, Y.-P. Hsu, and K.-T. Feng, "A POMDP-based spectrum handoff protocol for partially observable cognitive radio networks," 2009 IEEE Wireless Commun. Netw. Conf.

[21] L.-C. Wang, C.-W. Wang, and F. Adachi, "Load-balancing spectrum decision for cognitive radio networks," IEEE J. Sel. Areas Commun., vol. 29, no. 4, pp. 757-769, Apr. 2011

[22] C. R. Stevenson, G. Chouinard, Z. Lei, W. Hu, S. J. Shellhammer, and W. Caldwell, "IEEE 802.22: the first cognitive radio wireless regional area network standard," IEEE Commun. Mag., vol. 47, no. 1, pp. 130138, Jan. 2009.

[23] R. W. Wolff, "Poisson arrivals see time averages," Operations Research, vol. 30, no. 2, pp. 223-231, Mar./Apr. 1982

[24] X. Li and S. A. Zekavat, "Traffic pattern prediction and performance investigation for cognitive radio systems," 2008 IEEE Wireless Commun. Netw. Conf.

[25] C.-H. Ng and B.-H. Soong, Queueing Modelling Fundamentals with Applications in Communication Networks, 2nd edition. John Wiley \& Sons Inc., 2008.

[26] L. Kleinrock, Queueing Systems - Volume 2: Computer Applications. John Wiley \& Sons Inc., 1975.

[27] L.-C. Wang, C.-W. Wang, and C.-J. Chang, "Modeling and analysis for spectrum handoffs in cognitive radio networks," accepted by IEEE Trans. Mobile Comput., 2011.

[28] T. H. Cormen, C. E. Leiserson, R. L. Rivest, and C. Stein, Introduction to Algorithms. MIT Press and McGraw-Hill, 2001.

[29] C.-W. Wang, L.-C. Wang, and F. Adachi, "Performance gains for spectrum utilization in cognitive radio networks with spectrum handoff," 2009 International Symp. Wireless Personal Multimedia Commun.

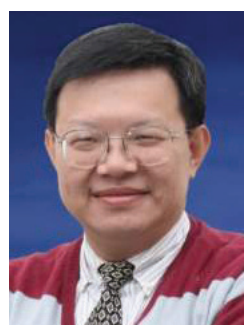

Li-Chun Wang (M'96 - SM'06 - F'11) received the B.S. degree from National Chiao Tung University, Taiwan, R. O. C. in 1986, the M.S. degree from National Taiwan University in 1988, and the Ms. Sci. and Ph.D. degrees from the Georgia Institute of Technology , Atlanta, in 1995, and 1996, respectively, all in electrical engineering. From 1990 to 1992, he was with the Telecommunications Laboratories of the Ministry of Transportations and Communications in Taiwan (currently the Telecom Labs of Chunghwa Telecom Co.). In 1995, he was affiliated with Bell Northern Research of Northern Telecom, Inc., Richardson, TX. From 1996 to 2000, he was with AT\&T Laboratories, where he was a Senior Technical Staff Member in the Wireless Communications Research Department. Since August 2000, he has joined the Department of Electrical and Computer Engineering of National Chiao Tung University in Taiwan and is the current Chairman of the same department. His current research interests are in the areas of radio resource management ,cross- layer optimized techniques for heterogeneous wireless networks, and cloud computing for mobile applications.
He was elected to the IEEE Fellow grade in 2011 for his contributions in cellular architectures and radio resource management in wireless networks. He also Distinguished Research Award of National Science Council, Taiwan in 2012, and was the co-recipient (with Gordon L. Stüber and Chin-Tau Lea) of the 1997 IEEE Jack Neubauer Best Paper Award for his paper "Architecture Design, Frequency Planning, and Performance Analysis for a Microcell/Macrocell Overlaying System," IEEE TRANSACTIONS ON Vehicular TECHNOLOGY, vol. 46, no. 4, pp. 836-848, 1997. He has published over 180 journal and international conference papers. He served as an Associate Editor for the IEEE TRANSACTIONS ON WIRELESS COMMUNICATIONS from 2001 to 2005, the Guest Editor of Special Issue on "Mobile Computing and Networking" for IEEE Journal on SELECTED AREAS IN COMMUNiCATIONS in 2005 and on "Radio Resource Management and Protocol Engineering in Future IEEE Broadband Networks" for IEEE Wireless Communications Magazine in 2006. He is holding nine US patents.

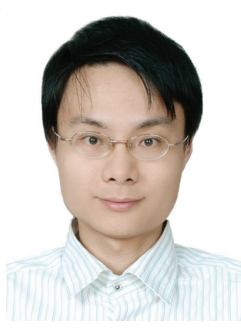

Chung-Wei Wang (S'07) received the B.S. degree in electrical engineering from Tamkang University, Taipei, Taiwan, in 2003, and the Minor M.S. and $\mathrm{Ph} . \mathrm{D}$. degrees in applied mathematics and communication engineering from the National Chiao Tung University, Hsinchu, Taiwan, in 2007 and 2010, respectively. From 2009 to 2010, he was also a visiting scholar in Tohoku University, Sendai, Japan. $\mathrm{He}$ was awarded the student travel grants from IEEE ICC 2009 and GLOBECOM 2010. He is currently working as a Principal Engineer at MStar Semiconductor, Inc., Taipei, Taiwan, and is responsible for the development of 3GPP technologies. His current research interests include cross-layer optimization, MAC protocols design, and radio resource management in cellular system, wireless sensor networks, ad hoc networks, and cognitive radio networks.

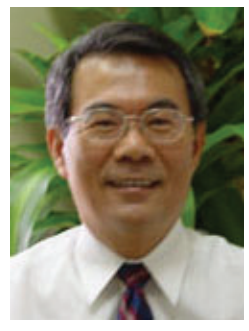

Chung-Ju Chang (F'08) was born in Taiwan, ROC, in August 1950. He received the B. E. and M. E. degrees in electronics engineering from National Chiao Tung University, Hsinchu, Taiwan, in 1972 and 1976, respectively, and the Ph.D. degree in electrical engineering from National Taiwan University, Taiwan, in 1985. From 1976 to 1988 , he was with Telecommunication Laboratories, Directorate General of Telecommunications, Ministry of Communications, Taiwan, as a Design Engineer, Supervisor, Project Manager, and then Managing Director. He also acted as a Science and Technology Advisor for the Minister of the Ministry of Communications from 1987 to 1989. In 1988, he joined the Faculty of the Department of Communication Engineering, College of Electrical Engineering and Computer Science, National Chiao Tung University, as an Associate Professor. He has been a Professor since 1993 and a Chair Professor since 2009. He was Director of the Institute of Communication Engineering from August 1993 to July 1995, Chairman of Department of Communication Engineering from August 1999 to July 2001, Dean of the Research and Development Office from August 2002 to July 2004, and Director of the Center for Information and Communications Research, Aim for Top University Plan sponsored by Ministry of Education from 2006 to 2010. Also, he was an Advisor for the Ministry of Education to promote the education of communication science and technologies for colleges and universities in Taiwan during 1995 - 1999. He was acting as a Committee Member of the Telecommunication Deliberate Body, Taiwan. Moreover, he serves as Editor for IEEE Communications Magazine and Associate Editor for IEEE TRANSACTIONS ON Vehicular TEChNOLOGY. His research interests include performance evaluation, radio resources management for cellular mobile communication systems, and traffic control for broadband networks. Dr. Chang is a member of the Chinese Institute of Electrical Engineers (CIEE) and a Fellow member of IEEE. 